\title{
Chrétien de Troyes (?), Guillaume d'Angleterre
}

\author{
Maria Colombo Timelli
}

\section{(2) OpenEdition}

\section{Journals}

\section{Édition électronique}

URL : http://journals.openedition.org/studifrancesi/8166

DOI : 10.4000/studifrancesi.8166

ISSN : 2427-5856

\section{Éditeur}

Rosenberg \& Sellier

\section{Édition imprimée}

Date de publication : 1 mai 2009

Pagination : 142-143

ISSN : 0039-2944

\section{Référence électronique}

Maria Colombo Timelli, «Chrétien de Troyes (?), Guillaume d'Angleterre », Studi Francesi [En ligne], 157

(LIII | I) | 2009, mis en ligne le 30 novembre 2015, consulté le 14 janvier 2021. URL : http://

journals.openedition.org/studifrancesi/8166 ; DOI : https://doi.org/10.4000/studifrancesi.8166

\section{Ce document a été généré automatiquement le 14 janvier 2021.}

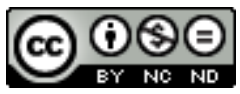

Studi Francesi è distribuita con Licenza Creative Commons Attribuzione - Non commerciale - Non opere derivate 4.0 Internazionale. 


\title{
Chrétien de Troyes (?), Guillaume d'Angleterre
}

\author{
Maria Colombo Timelli
}

\section{RÉFÉRENCE}

CHRÉTIEN DE TROYES (?), Guillaume d'Angleterre. Publication, traduction, présentation et notes par Christine FERLAMPIN-ACHER, Paris, Champion, 2007 ( «Champion Classiques Moyen Âge», 22), pp. 291.

1 Marqué par une double ambiguïté - quant à l'auteur et quant au genre littéraire Guillaume d'Angleterre jouit maintenant grâce à Christine Ferlampin-Acher d'une nouvelle édition/traduction dans une collection qui en permettra certainement une plus large diffusion.

2 Le roman est transmis par deux manuscrits seulement ( $C=$ Cambridge, Saint John's College, B9; $P$ = Paris, BnF, fr. 375), qui ont fourni, l'un ou l'autre, le texte base pour cinq éditions, les dernières en date étant celles d'Anthony J. Holden dans les TLF (1988, basée sur C) et celle d'Anne Berthelot pour la Pléiade (1994, basée sur P). Rappelons que l'édition collective dirigée par Michel Zink (Pochothèque, 1994) a en revanche exclu ce roman des œuvres de Chrétien de Troyes.

3 Comme le remarque l'éditrice, les appréciations sur Guillaume d'Angleterre varient sensiblement selon si l'on accepte ou non cette attribution, le «Crestiiens» du v. 1 ayant fait couler beaucoup d'encre; quant à elle, Christine Acher s'efforce de faire abstraction de cette hypothèse - qu'il lui paraît cependant prudent de décliner - pour offrir une analyse objective du roman sur le plan tant littéraire que stylistique. Elle discute ainsi les rapports certains que l'œuvre entretient avec les textes hagiographiques, en relevant presque en contrepoint les procédés de réécriture qui ramènent le récit vers le genre romanesque. Elle rappelle les thèmes majeurs (la chasse, avec le 'cor' qui constitue un objet emblématique, la question irrésolue de la faute initiale, le rapport entre Nature et Noreture), pour conclure sur une définition intelligente qui cherche à 
concilier les aspects parfois déroutants du récit: Guillaume d'Angleterre peut être lu comme une sorte d'«anthologie parodique», composée sans doute entre la fin du XII et le début $d u x{ }^{e}{ }^{e}$ siècle, inspirée certes par la légende de Saint Eustache, mais détournant certains éléments vers une lecture plus naïve sinon plus concrète. À ce propos, il me paraît intéressant de relever la portée du thème de l'argent, qui traverse le roman tout entier, incarné entre autres par les personnages des marchands (qui élèvent les jumeaux, accueillent le roi en exil, et seront enfin richement récompensés), mais présent aussi dans les listes d'objets, l'insistance sur leur valeur marchande etc.: un dépouillement des expressions relatives à ce champ sémantique amènerait sans doute à des découvertes intéressantes (ce qui, soit dit entre parenthèses, éloignerait encore davantage un tel récit de l'univers romanesque de Chrétien de Troyes).

L'Introduction comprend aussi une présentation, synthétique mais assez complète, des deux manuscrits $C$ et $P$ (celui-ci adopté comme manuscrit de base), manuscrits-recueils, le premier réunissant essentiellement des poèmes pieux, l'autre des romans en vers, entre autres Cligés et Erec et Enide (pp. 38-41). C.A. définit clairement les principes de son édition (pp. 42-44), qui se démarque des précédentes par des choix nettement conservatoires, ponctuellement commentés dans les notes. L'étude linguistique est en proportion très vaste (pp. 44-68): on soulignera l'intérêt des remarques sur la métrique, qui pose des problèmes de régularité (présence de nombreuses assonances, traitement souple de l'octosyllabe) rendus plus évidents par le nombre limité des corrections introduites. La Bibliographie rendra d'utiles services surtout pour la section spécialement consacrée à Guillaume d'Angleterre (pp. 70-73).

Dans l'ensemble, le texte est édité avec soin et la traduction est claire et élégante, ce qui fait regretter encore davantage un nombre non négligeable de fautes que l'on ne peut pas imputer toutes à une impression peut-être hâtive. Même en ignorant les coquilles, il faudra signaler: Lovel (lire: Marin, p. 9, 1. 11), en chasant (lire: en casant, p. 37, 1. 12), ë̈ (lire: uë, p. 44, l. 10), v. 1948 (lire: v. 1946, p. 44, l. 20; en outre, dans le vers cité, supprimer autres); à la p. 39, les ff. occupés par La vengeance de la mort d'Alexandre sont les 211-214, et non pas 208-210. Dans l'édition, qui occupe les pages de gauche, des corrections s'imposent: v. 58 (II: lire Il), p. 86, n. 6 (leçon de P?), v. 1703 (En'est il: supprimer l'apostrophe), v. 1832 (Contre s'est lui...: supprimer s'est), v. 2159 (le nombre .XXVIII. doit être remplacé par .XXIV.: cfr. l'apparat et la traduction). Quelques choix éditoriaux paraissent discutables: v. 628 (la leçon .XV., conservée, dépend selon toute probabilité d'une mauvaise lecture de .IV. [quatre dans C]); v. 1347 (D'une Nature, plutôt qu'en Bone Nature sur la base de $C$, aurait pu être modifié en Dame Nature, ainsi que C.A. elle-même le dit dans la note 95, p. 147). Pour ce qui est de la traduction, pages de droite, on remarque, en négligeant les coquilles: note 65, l. 2 (se sera: lire ne sera); la note 147 se rapporte au v. 2015 (et non pas 2009), la suivante au v. 2016 (et non pas 2015); dans la note 156, on lira «c'est sous un lit que le cor est trouvé» (et non pas: «sous un banc»); note 165: lire ceans (et non cheans); la note 170 se réfère au v. 2303 et doit par conséquent être rapportée à la traduction qui se lit p. 199, ligne 4 (Mais <tous> les quatre vents...); p. 203, ligne 2: lire «quelle que soit sa valeur» (et non pas «quelque soit»); p. 207, la traduction du «doit maine» (v. 2461) | «à son majeur» est étonnante, après ce qui est dit dans la note correspondante («Maine... est bien attesté au sens de 'petit'»). L'auteur de Guillaume d'Angleterre introduit de rares expressions imagées, entre autres pour signifier la valeur minimale des objets: «le vaillant d'une castaigne» (v. 157), «vaillant .I. festu» (v. 159), «vaillant une pume» (v. 2524); à ce sujet, les renvois à la thèse de Philippe 
Ménard (notes 20 et 179) pourraient être complétés par la référence au beau livre de Frankwalt Möhren, Le renforcement affectif de la négation par l'expression d'une valeur minimale en ancien français (Tübingen, Niemeyer, 1980).

6 L'édition est complétée par les apparats habituels: variantes significatives du ms. C (pp. 255-268), index des noms propres (pp. 269-270), glossaire sélectif (pp. 271-289).

7 J'ajouterai un relevé des expressions d'allure proverbiale et sentencieuse, qui se concentrent autour de trois noyaux: les affirmations morales, éventuellement d'origine évangélique («Qui s'umelie, si s'essauce, Ce dist on, et s'est verités», vv. 1024-25; «Car cols de lange ne fait plaie», v. 1570; «Mais tex fait mal, qui ne le set», v. 2956; «Mais qui pecce par ignorance N'i afiert pas grant peneance», vv. 3099-100), l'argent et la richesse («Qui rices est, moult troeve amis», v. 1574; «Que sage povre, hui est li jors, Tient on por fol en totes cors, Et rice fol tient on a sage», vv. 1581-83; «De povreté est lais mehains», v. 1972), et une condamnation presque sans appel des «vilains» («Vilains iriés est vif maufés», v. 1461; «En vilain a moult fole beste», v. 3219).

8 Reste à souhaiter que cette nouvelle édition suscite un regain d'intérêt pour Guillaume d'Angleterre, au-delà des questions de paternité de l'œuvre, qui a tout à gagner à être enfin lue de manière autonome, quel que soit son auteur. 